\section{Digital Subtraction Of Lipofuscin's Fluorescence}

Anda Cornea, Oregon Health Sciences University

Oregon Regional Primate Research Center corneaan@ohsu.edu

Fluorescence imaging of brains from older animals, particularly primates, can be hindered by the presence of lipofuscin, a strongly fluorescent pigment that accumulates with age, primarily in neuronal cells. Lipofuscin has a broad emission spectrum that overlaps emission of most fluorescent labels, is present throughout the brain and other tissues, and is very hard to quench chemically (Schnella et al., Journal of Histochemistry and Cytochemistry, Vol. 47, 719-730, 1999). A more convenient way to eliminate lipofuscin from fluorescence images is the use image arithmetic to subtract its signal. This method takes advantage of lipofuscin's broad emission spectrum that makes it appear in all fluorescence channels. Imaging an extra channel, for which there is no fluorescent label present, provides an image of lipofuscin alone (Figure 1).

Each channel provides an 8 bit monochromatic image. Contrast is adjusted so that lipofuscin has the same intensity in all three channels, and background is negligible, within noise values. As its intensity is usually much higher than that of fluorescent markers used, it is easy to saturate the signal given by lipofuscin and thereby ensure that it will indeed have the same intensity. Simple image subtraction will remove the contribution of lipofuscin from the initial image leaving the image of the fluorophore of interest (Figure 2):

Image $\mathrm{A}$ - Image $\mathrm{C}=$ Image $\mathrm{D}$

Image $B$ - Image $C=$ Image $E$
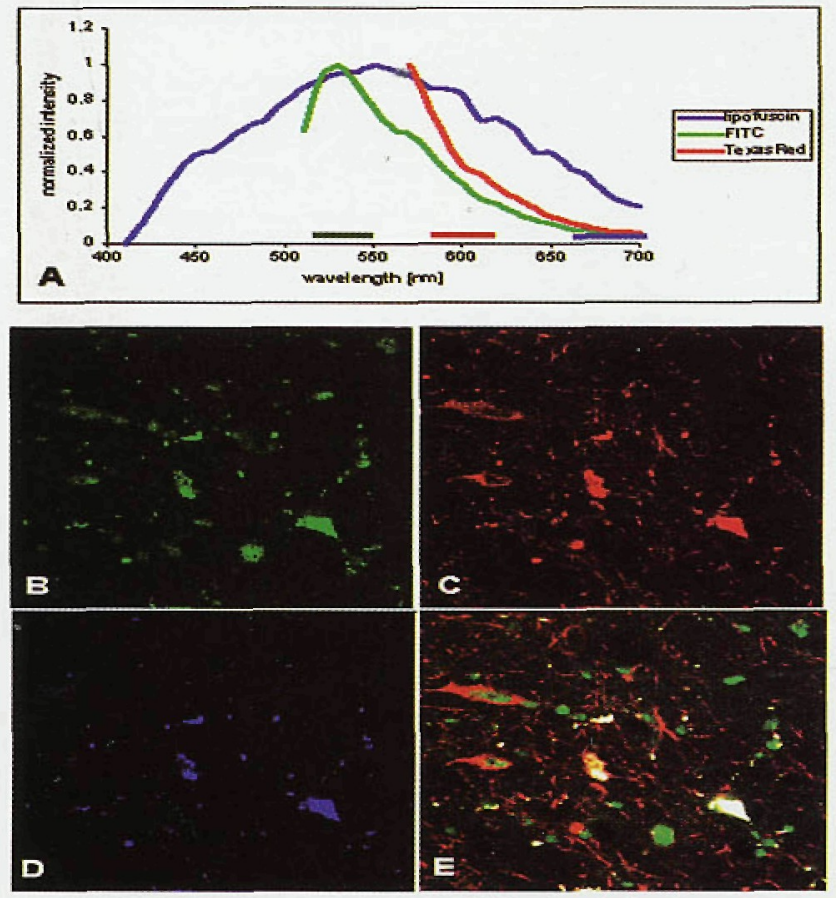

Figure 1: A. Emission spectrum of lipofuscin's fluorescence measured in situ using a Leica TSC SP confocal microscope with excitation at $361 \mathrm{~nm}$. Spectra for FITC and Texas Red were excited at $488 \mathrm{~nm}$ and $568 \mathrm{~nm}$ respectively. $B, C$, and D. images of a brain section labeled with FITC and Texas Red, excited with $488 \mathrm{~nm}$, and $568 \mathrm{~nm}$ and imaged in three channels with $30 \mathrm{~nm}$ spectral windows centered at $520 \mathrm{~nm}, 590 \mathrm{~nm}$ and $675 \mathrm{~nm}$ respectively. E. Overlay of A, B and C, showing lipofuscin in white.
In the case where acquisition of an additional channel is feasible, image arithmetic may still be used to remove lipofuscin it there is no significant overlap between at least two other fluoro: phores. In this case the image of lipofuscin alone can be obtained by the use of the logical $A N D$ operation between two chaniaels (Figure 3).

Image $\mathrm{C}=$ Image $\mathrm{A}$. AND. Image $\mathrm{B}$

Image $\mathrm{D}=$ Image $\mathrm{A}-$ Image $\mathrm{C}$

Image $E=$ Image $B$ - Image $C$

If there is some overlap at intensities lower than that of lipofuscin the AND operation may be applied to thresholded images.

Image arithmetic can be performed with the use of any of a number of available imaging software packages. Images Fresented here were processed with MetaMorph 4.5 (Universal Intag ing, West Chester, PA). An inexpensive (free) alternative is $\mathrm{NIH}$ Image (http://rsb.info.nih.gov/nih-image/).

Special thanks to Dr. Steve Kohama for kindly providing his migำ key brain specimens.

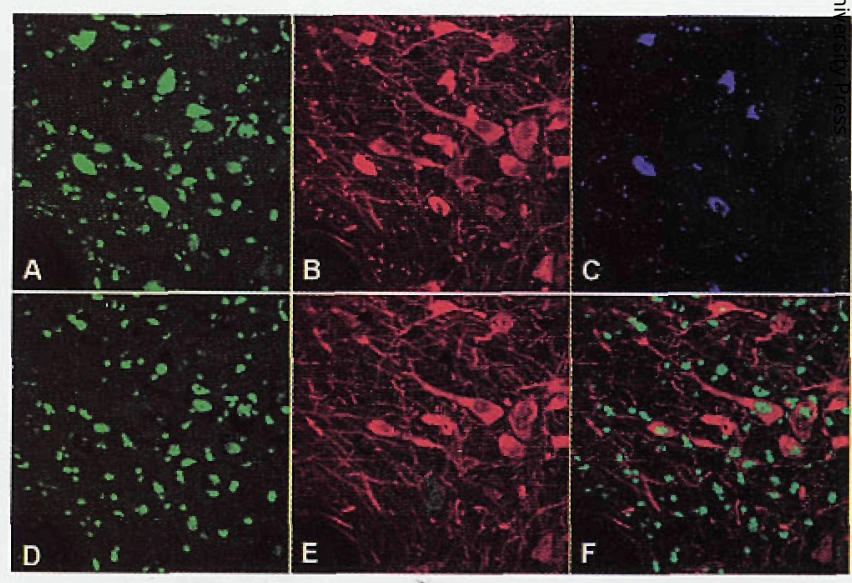

Figure 2: A. Image of FITC and lipofuscin, B. Image of Texas Red and lipofuscin, C. Image of lipofuscin, D. Image of FITC alone or $\mathrm{D}=\mathrm{A}-\mathrm{C}, \mathrm{E}$. Image of Texas Red alone or $\mathrm{E}=\mathrm{B}-\mathrm{C}, \mathrm{F}$. Overlay of $\mathrm{D}$ and $\mathrm{E}$ showing no lipofuscin.

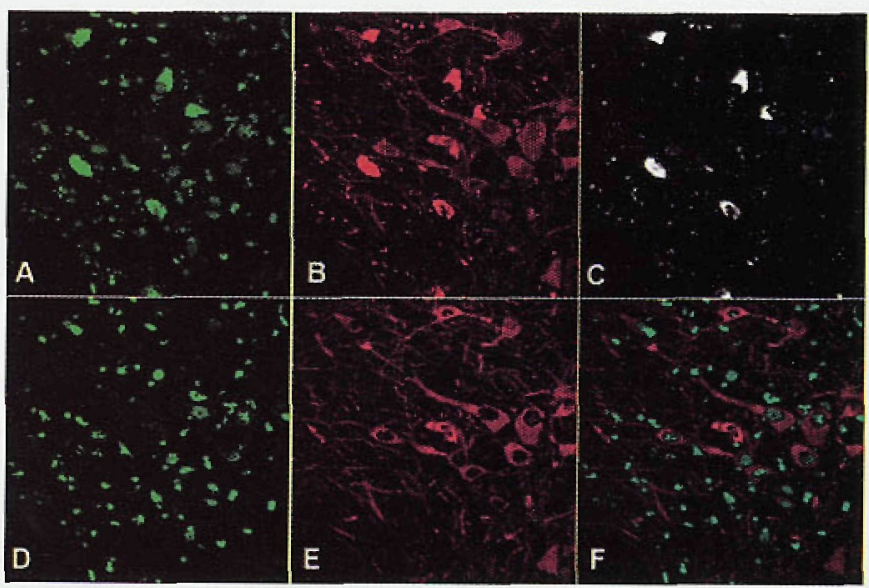

Figure 3. A. Image of FITC and lipofuscin, B. Image of Texas Red and lipofuscin. C. Image of A .AND. B, showing distribution of lipofuscin, D. Image of FITC alone or D=A-C, E. Image of Texas Red alone or $E=B-C, F$. Overlay of $D$ and $E$ showing no lipofuscin. 


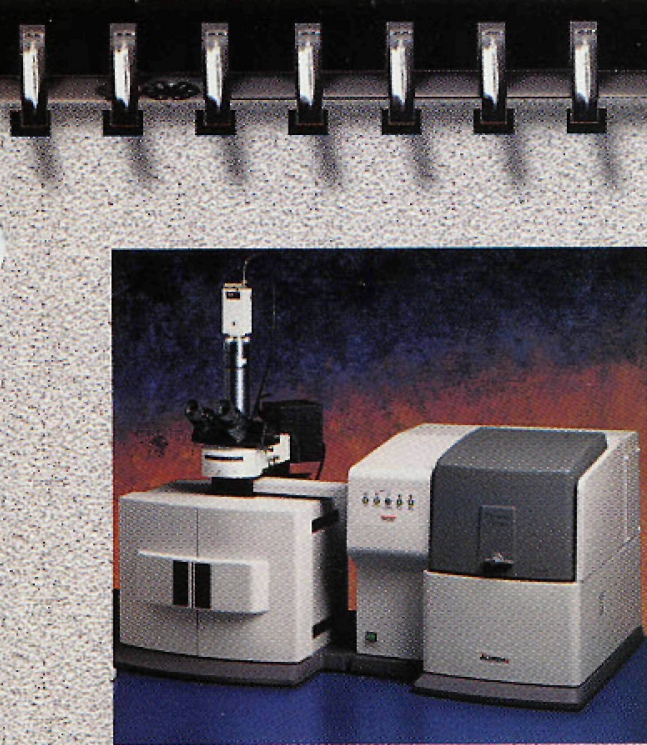

Almega ${ }^{i m}$ Dispersive Raman System

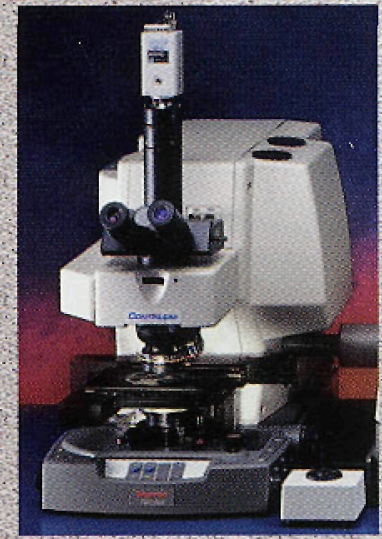

Continumm IR Microscope

\section{Unmistakably} family-with a personality all its own.

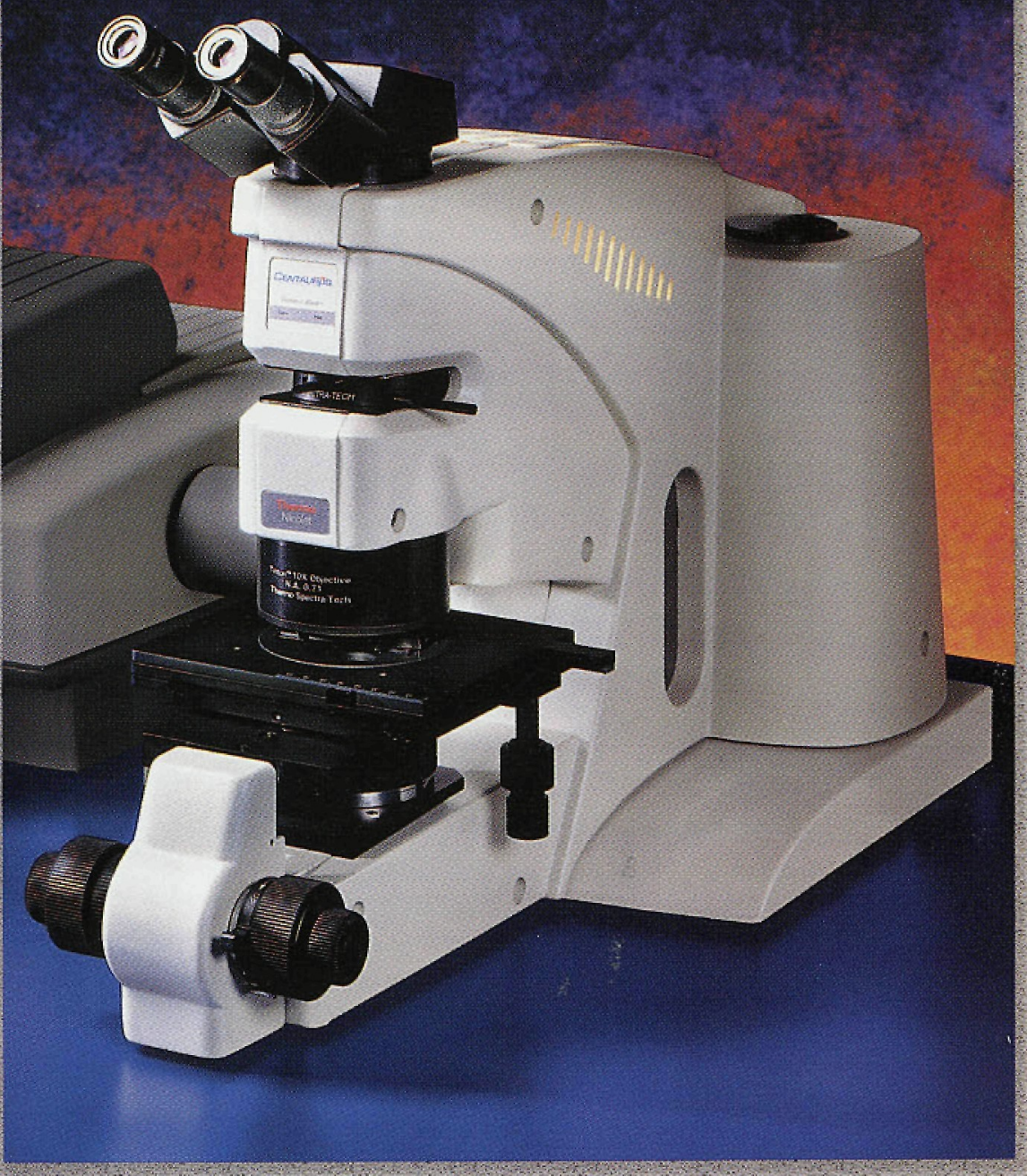

Meet our newest micro sampling family member, the Centaurus Tit IR microscope. At first glance, you'l notice it's compact and muttitalented: A closer inspection will also reveal its rugged workhorse persona.

The talented micro sampling family from Thermo Nicolet embraces both IR and Raman technologies. With such an impressive lineup, you'll find one instrument with the exact capabilities to meet your needs.

To see which member of our micro sampling family best suits your situation, visit www:thermonicolet.com. Better yet, call us. Together we'll make a perfect match. 